\title{
A Retrospective Study on Change in Morphology of Patellar Cartilage in Degenerative Disease of MRI
}

\author{
Ashraf Ahmed B ${ }^{1}$, Farooque MC ${ }^{2}$, Einstien $A^{3}$, Alex Daniel Prabhu ${ }^{4}$, Abubacker Sulaiman $F^{5}$, Parthasarathy E.A. ${ }^{6}$ \\ ${ }^{1}$ Associate Professor, Department of Radiodiagnosis, Chettinad Hospital and Research Institute, ${ }^{2}$ Final year Postgraduate, \\ Department of Radiodiagnosis, Chettinad Hospital and Research Institute, ${ }^{3}$ Associate Professor, Department of \\ Radiodiagnosis, Chettinad hospital and Research Institute, ${ }^{4}$ Professor, Department of Radiodiagnosis, Chettinad Hospital \\ and Research Institute, ${ }^{5}$ Professor, Department of Radiodiagnosis, Chettinad hospital and Research institute, ${ }^{6} \mathrm{Assistant}$ \\ Professor, Department of Radiodiagnosis, Chettinad Hospital and Research Institute, India
}

Corresponding author: Farooque MC, Final Year, Department of Radiology, Chettinad Hospital and Research Institute, Rajiv Gandhi Salai, Kelambakkam, Chennai - 603103, Tamilnadu, India

DOI: http://dx.doi.org/10.21276/ijcmsr.2019.4.3.13

How to cite this article: Ashraf Ahmed B, Farooque MC, Einstien A, Alex Daniel Prabhu, Abubacker Sulaiman F, Parthasarathy E.A.P. A retrospective study on change in morphology of patellar cartilage in degenerative disease of MRI. International Journal of Contemporary Medicine Surgery and Radiology. 2019;4(3):C58-C62.

\section{A B S T R A C T}

Introduction: Chondromalacia patellae are one of the most frequently encountered causes of anterior knee pain arising due to physical changes and biomechanical alterations. It is commonly observed among adolescents and females with idiopathic chondromalacia. The study aims to determine the presence of morphological changes in patellar cartilage and to analyze the assumption that aging results in thinning of patellar cartilage and that the amount of cartilage deformation among the elderly people differs from the younger age groups.

Material and methods: In the study, 50 patients with clinical diagnosis of anterior knee pain referred for MRI Knee Joint were randomly selected from the department of Radiology, Chettinad Health City and Research Institute, Kelambakkam. It is a retrospective, cross sectional study. The scanning was executed in a GE 1.5 tesla scanner with 8 Channel Knee array coil. Results: Out of the 50 patients who underwent MRI Knee Joint, 64\% (32 patients) were at or below 35 years of age while the rest $36 \%$ (18 patients) were above 35 years of age. The retrospective analysis revealed abnormal morphology of the patellar cartilage changes were higher in females i.e. $52 \%$ compared to male patients i.e. 48 patients.

Conclusion: MRI is a great tool in the imaging of various morphological differences of the patella and also in the evaluation of various cartilage pathologies.

Keywords: Change in Morphology, Patellar Cartilage, Degenerative Disease of MRI

\section{INTRODUCTION}

The term Chondromalacia patellae refers to a progressive course of softening of the articular cartilage present on the posterior surface of the patella which is characterized by fibrillation, thinning, focal protrusions, formation of ulcers, chondral defects and subchondral erosive changes. It is an anterior knee pain that arises due to the biomechanical and physical alterations in the patella. It is commonly referred to as the 'runner's knee' as it is predominantly observed among people involved in sports. It is also referred to as "patellar pain syndrome" by few authors. ${ }^{1,2}$

It is observed to be one of the prime causes of occurrence of anterior knee pain among the adolescents and females with idiopathic chondromalacia. The condition is observed to degenerate among the middle and older age groups. Patellofemoral pain syndrome (PFPS) is believed to be differential diagnosis of chondromalacia even though it is not considered to be under the umbrella term of PFPS. The other imaging methods used in diagnostic radiography are $\mathrm{x}$-ray and computed tomography $(\mathrm{CT})$. The main aim of the present study was to assess the presence of morphological changes in patellar cartilage among the 50 asymptomatic individuals and also to analyse that thinning of patellar cartilage occurs with aging and that elderly subjects display a different amount of cartilage deformation than do young subjects. ${ }^{3}$

A chondromalacia patella which is also known as CMP is inflammation of the underside of the patella and softening of the cartilage. It is a term sometimes treated synonymously with patellofemoral pain syndrome. Chondromalacia can be divided into 4 grades by MRI, typically using fat saturated proton density sequences. This grading system is the modified Outerbridge grading system, which was devised for arthroscopy initially for assessment of chondromalacia patella, but then modified and extended for all chondral surfaces. Grade I: focal areas of hyperintensity with normal contour arthroscopically: softening or swelling of cartilage. Grade II: blister-like swelling/fraying of articular cartilage extending to surface arthroscopically: fragmentation and fissuring within soft areas of articular cartilage. Grade III: partial thickness cartilage loss with focal ulceration arthroscopically: 
partial thickness cartilage loss with fibrillation (crab-meat appearance). Grade IV: full thickness cartilage loss with underlying bone reactive changes arthroscopically: cartilage destruction with exposed subchondral bone. ${ }^{4,5,6}$

Anterior knee pain is the most predominant clinical presentation chondromalacia patellae which aggravates through certain activities and sports such as running, stair climbing, and squatting that load the patellofemoral joint. The pain arising due to the condition restrains individuals from performing their routine and other physical activities. $^{7}$

MRI of the knee provides information that may assist the clinician in making appropriate decisions and also provide a roadmap for planning of surgical or other intervention procedures. It not only helps in detailed evaluation of osteoarthritis and other cartilage disorders in order to guide the surgeon to adopt the most appropriate strategy for the best feasible outcome but also helps to investigate the morphological parameters such as bone (cartilage interface area), thickness and volume of knee cartilages. ${ }^{8,9}$

Therefore, the aim of the present study was to assess the presence of morphological changes in patellar cartilage and also to evaluate that thinning of patellar cartilage occurs with aging and also elderly subjects display a different amount of cartilage deformation than do young subjects.

\section{MATERIALS AND METHODS}

The present study was a retrospective descriptive crosssectional study which was conducted in the Department of Radiology, Chettinad Health City and Research Centre, Kelambakkam. This study was carried out on 50 patients with a history of osteoarthiritis referred to Department of Radiology for MRI of knee and were randomly selected.

All data/images were collected from picture archiving communication system (PACS). All patients were imaged on 1.5 Tesla clinical scanner GE signa HDXT with dedicated extremity coils (surface coils) as both transmitter and receiver of radio frequency waves were applied. The imaging system is enclosed in a radio frequency room. For signal recording, an 8 Channel Knee array coil was used as provided by the manufacturer. Patients who underwent MRI Knee Joint with history of osteoarthritis were included in this study and patients on metallic implants, claustrophobic patients, early pregnancy, patients with impaired renal function and patients on cardiac pace maker were completely excluded from the study.

Magnetic resonance image can visualise joint structure directly and is recognised as a valid, accurate and reproducible tool to measure articular cartilage defects, volume thickness, and bone surface area.

\section{Anthropometry}

Weight was measured to the nearest $0.1 \mathrm{~kg}$ with shoes, socks and bulky clothes removed using a single pair of electronic scales which were calibrated using a known weight. Height was measured to the nearest $0.1 \mathrm{~cm}$ using a stadiometer. Knee pain was assessed by the questionnaire and was defined as pain for $>24$ hours in the past 12 months or daily pain on more than 30 days in the past year.

\section{Knee cartilage volume and thickness measurement}

An MRI scan of the right knee and left knee was performed. Knee cartilage volume and thickness for tibia and patella plates and tibia and patella bone sizes were determined. Medial and lateral tibial plateau bone area was determined by creating an isotropic volume from the three input images closet to the knee joint.

Different stages of the disease can be visualized through MRI which is as follows:

- $\quad$ Stage 1: The articular cartilage softens and swells as the vertical collagenous fibres break

- Stage 2: Separation of the superficial layer from the deep cartilaginous layer forms blisters in the articular cartilage

- Stage 3: This stage results in ulceration, fragmentation, fibrillation and fissures of cartilage that extends to the subchondral bone. This affects less than fifty percent of the patellar articular surface.

- Stage 4: More than fifty percent of the patellar articular surface gets affected at this stage resulting in eburnation and formation of crater. Sclerosis and erosions of the subchondral bone also occurs at this phase.

MRI Grading: Chondromalacia can be categorized into for types of grades using the fat saturated proton density sequences. This system is the modified version of the 'Outer bridge grading system' which was initially formulated for arthroscopy to assess chondromalacia patella; it was later modified for viewing all chondral surfaces.

\section{Grade I}

- Uses normal contour to display focal hyperintense areas

- Arthroscopically: Swelling and softening of the cartilage

- FS PD- weighted FSE images display focal areas of hyperintensity in the absence of any discontinuity in the smooth superficial cartilage contour. Early-stage blister formation with focal chondral convexity may also be observed.

\section{Grade II}

- Swelling of articular cartilage in the form of blister extends to the surface

- Arthroscopically: Fissuring and fragmentation of articular cartilage

- In addition to blister like swelling there may be fissuring and fragmentation confined the soft areas of articular cartilage. FS PD weighted demonstrates hyperintense superficial or deep fissures.

\section{Grade III}

- Partial loss of surface cartilage thickness within the focal areas of ulceration

- Arthroscopically: Crab-meat appearance due to partial loss of cartilage thickness with fibrillation

- On FSPD-weighted FSE images, the absorbed fluid on the affected articular cartilage surface is seen as highsignal-intensity sites. Axial or sagittal FS PD FSE scans are also helpful in identification of focal ulcerations and "crabmeat" lesions. Basal degeneration may be seen in association with surface fibrillation in grade 3 .

\section{Grade IV}

- Due to underlying bone reactive changes, full thickness 
of the cartilage is lost

- Arthroscopically: Exposure of subchondral bone due to destruction of the cartilage

- The destruction of articular cartilage and the underlying fluid represents ulceration and exposure of subchondral bone on MR images

\section{STATISTICAL ANALYSIS}

Data was entered in Microsoft excel sheet and analysis was done through Mann Whitney U test with the help of SPSS software version 19 .

\section{RESULTS}

In this study, 50 patients presented with history of osteoarthiritis were randomly selected. The intention of the study was to determine the presence of morphological changes in patellar cartilage among various age groups. Table no. 1 shows distribution of patients with chondromalacia based on gender. The retrospective analysis of 50 patients who underwent MRI Knee Joint revealed that abnormal morphology of the patellar cartilage changes were more common among females (52\%) as compared to males (48\%). Table no. 2 shows distribution of patients with

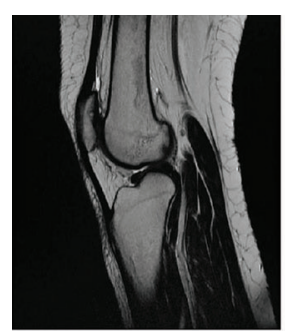

Sagittal T2PD FAT SAT

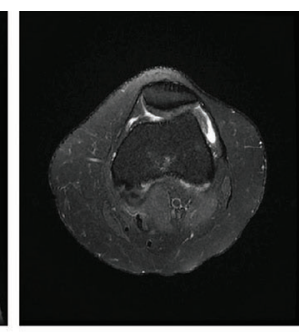

Axial PD FAT SAT

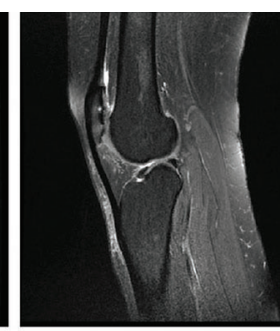

Sagittal PD FAT SAT
Figure-1: Shows Grade I Chondromalacia Patella in MRI Knee

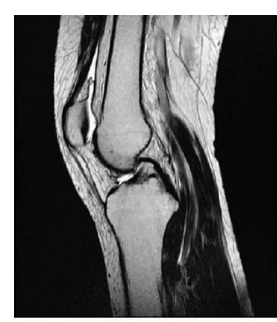

Sagittal T2PD FAT SAT

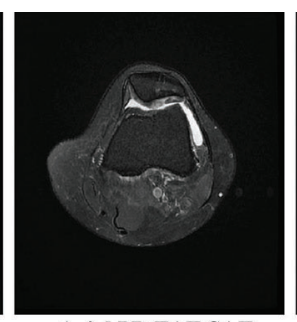

Axial PD FAT SAT

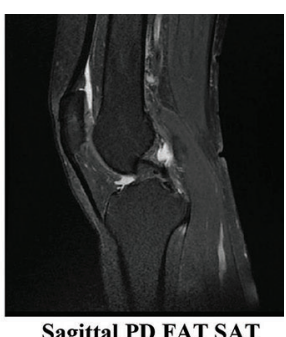

Sagittal PD FAT SAT
Figure-2: Shows Grade II Chondromalacia Patella in MRI Knee

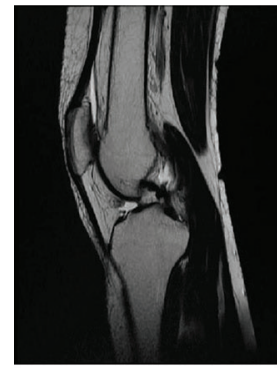

Sagittal T2 PD FAT SAT

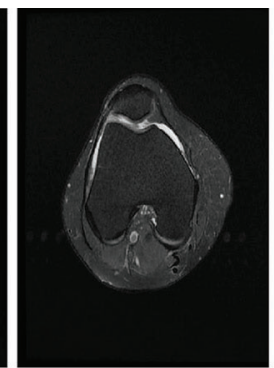

Axial PD FAT SAT

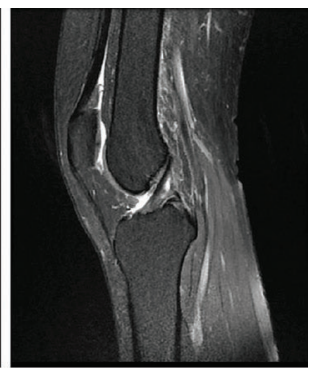

Sagittal PD FAT SAT
Figure-3: Shows Grade III Chondromalacia Patella in MRI Knee

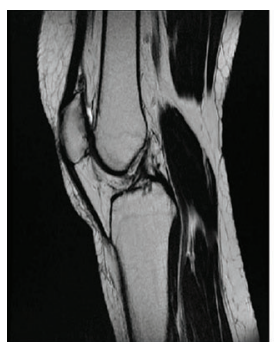

Sagittal T2 PD FAT SAT

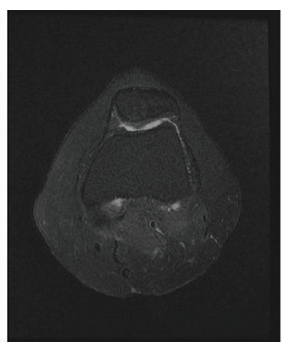

Axial PD FAT SAT

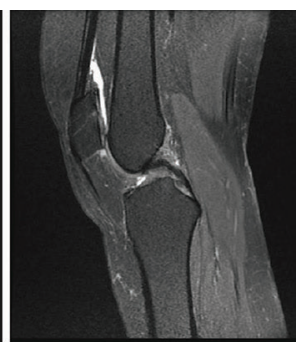

Sagittal PD FAT SAT
Figure-4: Shows Grade IV Chondromalacia Patella in MRI Knee

\begin{tabular}{|l|l|l|}
\hline Gender & Number of patients & Percentage \\
\hline Male & 24 & $48 \%$ \\
\hline Female & 26 & $52 \%$ \\
\hline Table-1: Shows distribution of patients with Chondromalacia \\
based on gender \\
\hline
\end{tabular}

\begin{tabular}{|l|c|c|}
\hline Age & Number of patients & Percentage \\
\hline$\leq 25$ Years & 11 & $26 \%$ \\
\hline $26-35$ & 19 & $38 \%$ \\
\hline Total $(<35)$ & 30 & $64 \%$ \\
\hline $36-45$ & 8 & $16 \%$ \\
\hline$>45$ Years & 12 & $20 \%$ \\
\hline Total $(>35)$ & 20 & $36 \%$ \\
\hline
\end{tabular}

Table-2: Shows distribution of patients with Chondromalacia based on age

\begin{tabular}{|c|c|c|c|c|c|c|}
\hline \multicolumn{5}{|l|}{ Knee side } & \multicolumn{2}{|c|}{$\begin{array}{c}\text { Mann Whitney } \\
\text { Test }\end{array}$} \\
\hline \multicolumn{3}{|l|}{ Right } & \multicolumn{2}{|c|}{ Left } & \multirow[t]{2}{*}{ U value } & \multirow[t]{2}{*}{$p$ value } \\
\hline Age group & No. & $\%$ & No. & $\%$ & & \\
\hline$\leq 25$ & 4 & $16 \%$ & 9 & $36 \%$ & \multirow{4}{*}{205.000} & \multirow{4}{*}{0.029} \\
\hline $26-35$ & 9 & $36 \%$ & 10 & $40 \%$ & & \\
\hline $36-45$ & 4 & $16 \%$ & 4 & $16 \%$ & & \\
\hline$>45$ & 8 & $32 \%$ & 2 & $8 \%$ & & \\
\hline
\end{tabular}

chondromalacia based on age It was also observed that the frequency of occurrence of chondromalacia was more among the younger age group below 35 years i.e. $64 \%$ in comparison to the elder subjects i.e. $36 \%$. Furthermore, it was found that the difference between the knee sides based on the age group using Mann Whitney test was also found to be statistically significant at $p$ value $=0.029$ and $u$ value at $=205.000$. The study indicated that abnormal morphology of the patellar cartilage changes were more common among females as compared to males and the frequency of occurrence of chondromalacia was more among the younger age group.

Figure no. 1 showed a case of 32 years old female with history of minor swelling in knee with Grade I chondromalacia patella in MRI knee. Figure no. 2 showed a case of 27 years old female with history of tenderness in knee with Grade II chondromalacia patella in MRI knee. Figure no. 3 showed a case of 30 years old female with history of pain after prolonged rest has showed Grade III chondromalacia 
patella in MRI knee. Figure no. 4 showed a case of 34 years old female with history of anterior knee pain with Grade IV chondromalacia patella in MRI knee.

\section{DISCUSSION}

In the present study, 50 patients with clinical diagnosis of anterior knee pain were referred for MR imaging in Chettinad Health City and Research Institute, Kelambakkam. The MRI Knee Joint was performed in GE 1.5 Tesla scanner with 8 Channel Knee array coil. The study intended to determine the changes in morphology of patellar cartilage in degenerative diseases through MRI. ${ }^{10}$

From previous literature, it was found that the compartments of the knee joint cartilage of the elderly population were different as compared to the younger age groups. Chondromalacia patellae is considered to be one of the prime cause of anterior knee pain arising due to the physical and biomechanical changes common among adolescents and females with idiopathic chondromalacia; the condition degenerates with aging. ${ }^{11}$

Some common causes of acute chondromalacia included fracture, instability and direct trauma. While that of chronic chondromalacia included quadricep imbalance, subluxation, post traumatic mal-alignment, increased quadriceps angle, excessive lateral pressure syndrome and PCL injuries and in some cases also results from inflammatory arthritis, synovitis and infection. Articular cartilage plays an important role in the distribution of contact loads within the patellofemoral joint. It has been suggested that thinner cartilage experiences higher peak stresses when compared with thicker cartilage under the same loading conditions. ${ }^{12}$

The MR sequences that have been most commonly used for cartilage quantification over recent years have been fatsupressed T1-weighted spoiled gradient echo. Different MRI vendors name these sequences differently (FLASH= fast low angle shot, SPGR= spoiled gradient recalled acquisition at steady state), but they are otherwise very similar and are available on most whole-body clinical MRI systems with $\geq 1.0$ Tesla (T) magnetic field strength. Studies on morphological assessment of articular cartilage with MR imaging of whole-body scanners have the advantage of lower installation and management costs as compared with wholebody systems. ${ }^{13}$

Chondral lesions are diagnosed through signal, thickness and morphological abnormalities of the hyaline cartilage. The most specific MRI signal for chondral lesions is the presence of areas of greater signal in proton density and T2-weighted sequences and on the echo gradient. However, this signal does not have the sensitivity to determine lesion presence. A study demonstrated that $70 \%$ of chondral lesions presented a high signal in relation to normal cartilage on the proton density sequence, while $20 \%$ presented a signal similar to that of normal cartilage (i.e. lesions were not seen on $\mathrm{MRI}$ ) and $10 \%$ presented a low signal in relation to normal cartilage. It was also demonstrated that high-signal lesions were more extensive than were lesions with the same signal or low signal. ${ }^{14}$

Chronic chondral lesions with detachment of cartilaginous fragments into the joint lead to chronic irritation of the synovium and may cause synovitis. In some cases, the synovial response is very extensive and may take on a pseudotumoral appearance on imaging examinations. It is also common for there to be free bodies in the joints, especially in the suprapatellar recesses, posteriorly to the femorotibial and popliteal joints. ${ }^{15}$

\section{CONCLUSION}

Magnetic Resonance Imaging was a very effective imaging modality in the evaluation of cartilage and also in the characterization of various pathologies of the cartilage. Various advancements in magnetic resonance imaging also allowed in assessing and quantifying the thickness and volume of the cartilage. It also helped in physiological evaluation of changes in the composition of proteoglycan content and collagen ultra-structure. Hence, MRI is a great tool in the imaging of various morphological differences of the patella and also in the evaluation of various cartilage pathologies.

\section{REFERENCES}

1. Buckwalter JA, Mankin HJ. Articular cartilage: tissue design and chondrocytematrix interactions. Instr Course Lect. 1998; 47(1):477-86.

2. Recht MP, Resnick D. Magnetic resonance imaging of articular cartilage: an overview. Top Magn Reson Imaging. 1998; 9(6):328-36.

3. Hodler J, Resnick D. Current status of imaging of articular cartilage. Skeletal Radiol. 1996; 25(8):703-9.

4. Disler DG, McCauley TR. Clinical magnetic resonance imaging of articular cartilage. Top Magn Reson Imaging. 1998; 9(6):360-76.

5. Rubenstein JD, Li JG, Majumdar S, Henkelman RM. Image resolution and signal-to-noise ratio requirements for MR imaging of degenerative cartilage. AJR Am J Roentgenol. 1997; 169(4):1089-96.

6. Schaefer FK, Kurz B, Schaefer PJ, Fuerst M, Hedderich J, Graessner J, et al. Accuracy and precision in the detection of articular cartilage lesions using magnetic resonance imaging at 1.5 Tesla in an in vitro study with orthopedic and histopathologic correlation. Acta Radiol. 2007; 48(10):1131-7.

7. Mohr A. The value of water-excitation 3D FLASH and fat-saturated PDw TSE MR imaging for detecting and grading articular cartilage lesions of the knee. Skeletal Radiol. 2003; 32(7):396-402.

8. Potter HG, Linklater JM, Allen AA, Hannafin JA, Haas SB. Magnetic resonance imaging of articular cartilage in the knee. An evaluation with use of fast-spin-echo imaging. J Bone Joint Surg Am. 1998; 80(9):1276-84.

9. Patil Prakash, Adke Shrishail, Shaha Pramod, Bhoite Amol, Kumar Snehil. A cross-sectional study to evaluate role of magnetic resonance imaging in ligament injuries of knee joint in a tertiary healthcare institute. International Journal of Contemporary Medical Research. 2018; 5(8):1-4.

10. Cicuttini F, Forbes A, Asbeutah A, Morris K, Stuckey S. Comparison and reproducibility of fast and conventional spoiled gradient-echo magnetic resonance sequences in the determination of knee cartilage volume. J Orthop Res. 2000; 18(4):580-4. 
11. Burstein D, Bashir A, Gray ML. MRI techniques in early stages of cartilage disease. Invest Radiol. 2000; 35(10):622-38.

12. Manek NJ, Lane NE. Osteoarthritis: current concepts in diagnosis and management. Am Fam Physician 2000; 61(7):1795-804.

13. Blumenkrantz G, Majumdar S. Quantitative magnetic resonance imaging of articular cartilage in osteoarthritis. Eur Cell Mater 2007; 13(2):76-86.

14. Eckstein F, Burstein D, Link TM. Quantitative MRI of cartilage and bone: degenerative changes in osteoarthritis. NMR Biomed 2006; 19(6):822-54.

15. Gray ML, Burstein D, Xia Y. Biochemical (and functional) imaging of articular cartilage. Semin Musculoskelet Radiol 2001; 5(1):329-43.

Source of Support: Nil; Conflict of Interest: None

Submitted: 28-02-2019; Accepted: 24-03-2019; Published online: 06-08-2019 